\title{
Advanced methods for the characterization of few-cycle light pulses: a comparison
}

\author{
Gero Stibenz ${ }^{1}$, Claus Ropers ${ }^{1}$, Christoph Lienau ${ }^{1}$, Christian Warmuth ${ }^{2}$, Adam S. Wyatt ${ }^{3}$, \\ Ian A. Walmsley ${ }^{3}$, Günter Steinmeyer ${ }^{1}$ \\ 1 Max-Born-Institut für Nichtlineare Optik und Ultrakurzzeitspektroskopie, Max-Born-Straße 2a, D-12489 Berlin, Germany \\ 2 FEMTOLASERS Produktions GmbH, Fernkorngasse 10, A-1100 Vienna, Austria \\ 3 Clarendon Laboratory, University of Oxford, Parks Road, Oxford, OX1 3PU, United Kingdom
}

Received: date / Revised version: date

\begin{abstract}
We discuss and compare four methods for measuring the width and pulse profile of ultrashort pulses. For our comparison, we use stable sub-7-fs pulses from a Ti:sapphire oscillator. Interferometric autocorrelation, spectral phase interferometry for direct electric-field reconstruction (SPIDER), a spatially-encoded variant of SPIDER, and interferometric frequency-resolved optical gating (IFROG) are utilized for characterizing pulses from the oscillator. The methods are found to agree within $5 \%$ as far as determination of the pulse width is concerned. However, differences are observed in the satellite structure reconstructed by either method. The current state of the art of measuring ultrashort pulses with these methods is reviewed and current limitations, in particular for characterizing complex pulse shapes, are discussed.
\end{abstract}

PACS: 06.60.Jn, 07.60.Ly, 42.65.Re, 42.79.Hp

\section{Introduction}

The characterization of femtosecond light pulses has experienced major breakthroughs during the last decade. While the traditional autocorrelation techniques can neither provide the pulse shape nor its exact duration, techniques such as frequency-resolved optical gating (FROG, [1-3]) and spectral phase interferometry for direct electric-field reconstruction (SPIDER, [4,5]) do not only measure the exact temporal pulse profile but also the corresponding phase. These techniques render a comprehensive picture of the pulse shape, either in the temporal or, alternatively, in the spectral domain. Therefore such techniques are also referred to as complete characterization methods. One major advantage of the complete methods is that they immediately reveal what kind of distortion causes temporal broadening of a laser pulse. Dispersive broadening, for example, is readily identified as a positive curvature of the spectral phase. This is a particular advantage in the sub-10fs range, where pulse shapes often deviate significantly from simple model functions such as the hyperbolic secant shape. In contrast, the lack of information on the pulse shape in traditional autocorrelation results in a rather broad range of pulse durations being compatible with the measured data, which often resulted in a systematic underestimation of the pulse width for such short pulses.

Unfortunately the use of complete methods becomes increasingly challenging with decreasing pulse duration. One generally encountered problem is the enormous bandwidth of few-cycle pulses [6,7]. As all characterization techniques employ a nonlinear optical process, a natural limit is reached when the nonlinear conversion products start to overlap with the fundamental spectrum. For second-harmonic ( $\mathrm{SH}$ ) based techniques such as the interferometric autocorrelation (IAC, [8]) and SHFROG, this limit is reached for octave spanning spectra. Being based on the nonlinear interaction of a narrowband signal and a broadband signal, SPIDER can be pushed to slightly wider coverage. A more technical constraint is the large bandwidth of the nonlinear conversion process, which requires very thin optical crystals of less than $10 \mu \mathrm{m}$ thickness. Also, the considerable complexity of the pulse shapes in the sub-10-fs regime, often consisting of several well-separated peaks in either domain, causes significant problems. When a certain contrast ratio is exceeded in the pulse structure, the detection method may loose track of the coherence between different spectral or temporal portions of the wave packet, which then results in an erroneous reconstruction of the relative phase delay between these portions [9]. Measures to extend the dynamic range of pulse characterization techniques and to avoid this artifact have been discussed [10].

Other problems are more specific to a particular technique. FROG, for example, often makes use of a non- 
collinear autocorrelation geometry. For a good reason, this geometry was always avoided in ultrashort-pulse measurements using the traditional IAC as geometric beam-smearing limits the temporal resolution in such a noncollinear set-up $[11,12]$. These effects become severe for pulses of about 2 optical cycles duration $(5.5 \mathrm{fs}$ at $800 \mathrm{~nm}$ center wavelength). Different measures have been discussed to reduce or avoid this problem, such as aperturing [12], a type-II collinear geometry [13], and also interferometric FROG [14]. SPIDER, on the other hand, may have some difficulties in characterizing complex pulse satellite structures as it essentially relies on sampling the spectral phase of a pulse with a limited number of points. A recently developed variant of SPIDER, Spatially Encoded Arrangement for SPIDER (SEA-SPIDER) may be an advantageous alternative for such complex pulses [15]. As this method is based on detection of a two-dimensional array rather than only a single spectrum, it makes use of a much larger amount of data that allows for the differentiation of a much wider variety of pulse shapes. SEA-SPIDER also alleviates the requirement of a high spectral resolution for dense sampling of the spectral phase.

The wealth of potential artifacts and pitfalls in the sub-10-fs regime calls for some guidance. In particular, it is interesting to compare the performance of different advanced techniques for pulse characterization in this difficult regime. For this purpose, we selected four methods and used them to characterize pulses from a commercial Ti:sapphire oscillator with less than $7 \mathrm{fs}$ pulse duration. This laser was chosen because it combines a pulse duration corresponding to only 2.5 optical cycles with an excellent long-term stability, which allowed for measurements on three consecutive days. As characterization methods we used the SPIDER method, interferometric FROG, and SEA-SPIDER. For comparison we also applied traditional IAC to characterize this laser.

\section{Experimental setups}

For a systematic comparison of different characterization methods, we used a Femtolasers "Rainbow" oscillator. This laser is specified to deliver $>3.8 \mathrm{~nJ}$ pulses at below $7 \mathrm{fs}$ pulse duration. The repetition rate of the laser is $80 \mathrm{MHz}$, i.e., it delivers a cw output power of $300 \mathrm{~mW}$. The laser is pumped by an all-solid-state green pump laser (Spectra Physics Millennia), which leads to an excellent noise performance. Moreover, the mechanical construction of the oscillator incorporates a temperature stabilized baseplate. The mechanical design is nearly monolithic and widely avoids the use of adjustable roller-bearing stages, which results in minimal drift over extended periods of time. Consequently, we observed nearly identical mode-locked spectra on three consecutive days, which were used for the measurements described below.

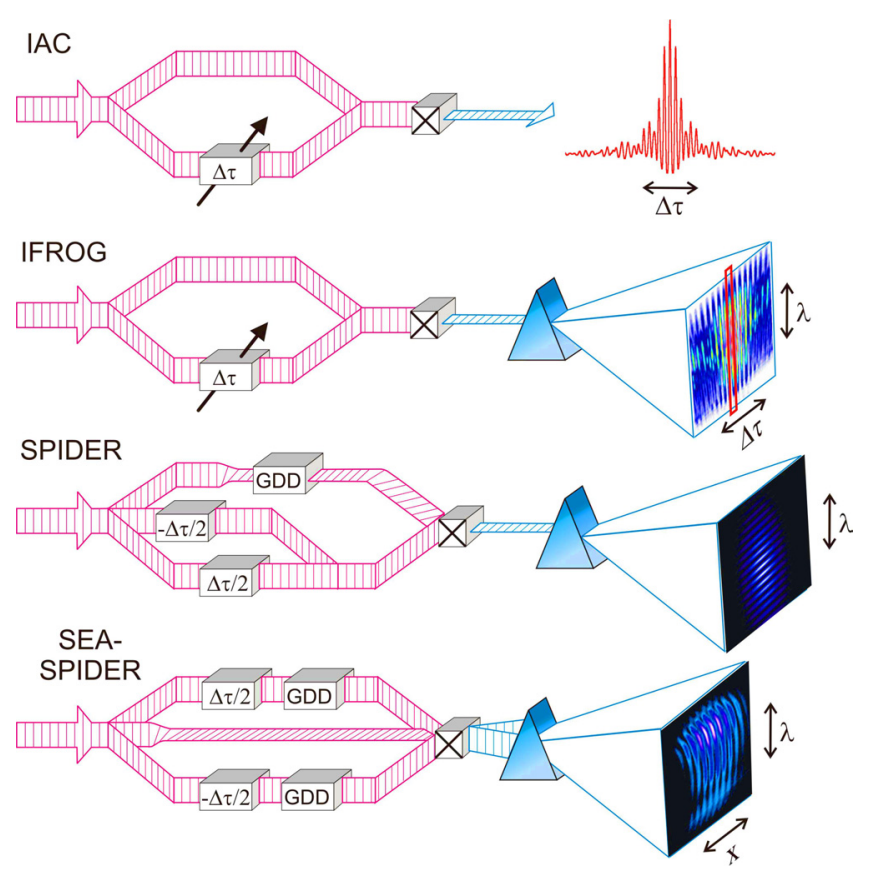

Fig. 1 Schematic representation of the setups used for characterizing few-cycle pulses. Both, correlation-based and spectral-interferometry based methods have been applied. The former rely on a Michelson interferometer with an adjustable delay $\Delta \tau$ between the two arms. The interferometric autocorrelation (IAC) records the spectrally integrated signal, whereas interferometric FROG (IFROG) additionally spectrally resolves it. The two SPIDER variants employ a non-collinear interaction of up to three beams with fixed time delays. All but one of these beams have experienced a substantial amount of group delay dispersion (GDD), which makes their interaction quasi-cw. Traditional SPIDER records only one-dimensional spectral information, whereas SEA-SPIDER requires a two-dimensional detection system.

This laser oscillator was analyzed with three different advanced methods for pulse characterization, namely IFROG, SPIDER, and SEA-SPIDER. Additionally, we also measured the interferometric autocorrelation of the pulses, mainly for comparison with calculated interferometric autocorrelations from the result of the three other methods. The setups of the different characterization methods are schematically compared in Fig. 1. For a more detailed description of the individual methods the reader is referred to the original literature, in particular Refs. $[3,5,16]$. For the implementation of the setups, all possible measures were taken to ensure sufficient bandwidth for the characterization of pulses down to $5 \mathrm{fs}$ duration. A particular concern are beam splitting schemes, which were either implemented using inconel coated metallic beam splitters or making use of a Fresnel surface reflection. The Michelson-type beam splitting arrangements described in the following have always been designed to be fully balanced, i.e., each of the pulses experiences the same number of reflections and transmissions and the same amount of dispersive mate- 
rial. Wherever possible, all-reflective optics were used, in particular for focusing into the nonlinear optical crystals. For the beam splitter mirrors we nearly exclusively used substrates of $1 \mathrm{~mm}$ thickness, which turn out to be a good compromise between spatial beam distortion due to bending of the substrates and dispersive distortion of the temporal pulse profile. Furthermore, we used very thin $\mathrm{BBO}$ crystals of a few 10 microns thickness. For the IFROG method and the interferometric autorcorrelation, where bandwidth limitation is a particular concern, the thinnest 10-micron thick BBO crystals commercially available were used.

A further measure is necessary to ensure comparability of the different methods. With pulses shorter than $10 \mathrm{fs}$, dispersive broadening in air already starts to play a role for path length differences of a few $10 \mathrm{~cm}$. Quite obviously, it is far from simple to set up four different characterization methods with the additional constraint of identical path lengths into the setup. Additionally, as will be discussed below, the spectral-interferometry based methods account for dispersion in the beam splitting scheme or avoid it in the first place. The correlationbased counterparts, in contrast, always add dispersion in their beam splitter substrates. Therefore, we chose to carefully measure path length differences and also account for intrinsic dispersion of the setups. As a reference point we selected the input of the SPIDER set-up and optimized the laser to yield the shortest pulse at this position. All other measurements were corrected accordingly as if they were measured at the same position and as if their intrinsic dispersion was zero. As such a simple correction is not possible for the IAC, the autocorrelator was placed closer to the laser to compensate for the dispersion of its $0.5 \mathrm{~mm}$ beam splitter substrates, which correspond to an equivalent air path of approximately $1 \mathrm{~m}$.

\section{Interferometric Autocorrelation}

Figure 2 shows an interferometric autocorrelation, which was measured with a commercial device (Femtolasers Femtometer) that was designed according to the general guidelines discussed above. We use a single sweep of the autocorrelator to avoid drift problems of the interferometer. The gray line in Fig. 2 depicts the measured IAC signal. This data was cleaned from high-frequency noise components by Fourier filtering. We find excellent symmetry of the signal and a ratio between the far wings and the peak of the signal of 1:7.5, i.e., close to the ideal value of 1:8. From the measurement of the laser spectrum, we determine a center wavelength of $806 \mathrm{~nm}$, which we use to identify the fundamental periodicity of the IAC trace for calibrating the delay axis. This value of $806 \mathrm{~nm}$ was independently checked in the FROG measurements by finding the proper frequency shift to match the FROG frequency marginal and the autocorrelation of the fundamental pulse spectrum. The carrier frequency is then

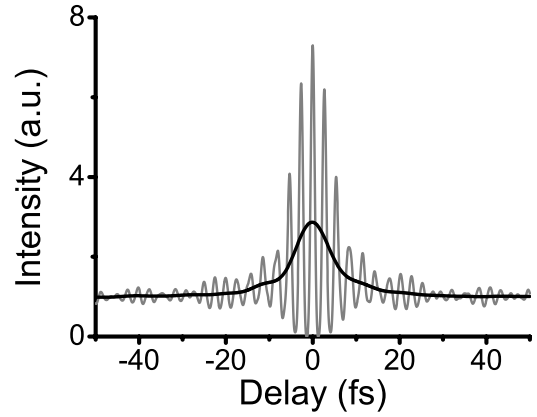

Fig. 2 Measured Autocorrelation: interferometric autocorrelation (IAC, gray line) and intensity autocorrelation with background (AC, black line). The AC is retrieved by Fourier filtering the DC-part of the IAC. The FWHM of the AC is $10.03 \mathrm{fs}$.

localized in the Fourier transform of the IAC signal at the position of the center of gravity of the first modulation sideband. Fourier filtering the central band around zero frequency allows for extraction of the unmodulated intensity autocorrelation (AC) on top of a SHG background. The resulting curve is shown as a black line in Fig. 2. The full width at half maximum (FWHM) of this $\mathrm{AC}$ amounts to $10.0 \mathrm{fs}$, which yields a pulse duration of $6.5 \mathrm{fs}$ assuming a hyperbolic secant pulse shape. As a different and less biased method, we also used a second method for evaluation of the pulse duration. Starting from the transform limit ( $5.2 \mathrm{fs}$ FWHM), we adjusted the chirp of the pulse to achieve an optimum fit between measured and calculated autocorrelation for the central part of the trace. This method yielded a duration of $6.3 \mathrm{fs}$, which is in remarkably good agreement with the hyperbolic secant evaluation. For either method of estimating the pulse duration, deviations are discernible as small satellite pulses at around \pm 10 fs and \pm 20 fs delay. We did not employ iterative fitting algorithms to reconstruct the pulse intensity and phase profile from the combination of IAC and power spectrum (see e.g. Refs. $[12,17])$ as an unambiguous reconstruction is not possible from the experimental data [18]. However, as will be shown below, the IAC can serve as an independent and sensitive marginal check for the complete characterization techniques.

\section{Traditional SPIDER}

In our experiments we used an SPIDER apparatus that combines few-cycle temporal resolution with at a relatively high detection sensitivity [10]. The latter is accomplished by an optimized beam splitting scheme that consists of a single fused silica etalon of $150 \mu \mathrm{m}$ thickness. The etalon thickness was chosen to yield a rather small modulation period of the interferogram corresponding to less than 5 pixels on the camera. We use a modulation period close to the sampling limit of the camera, because 


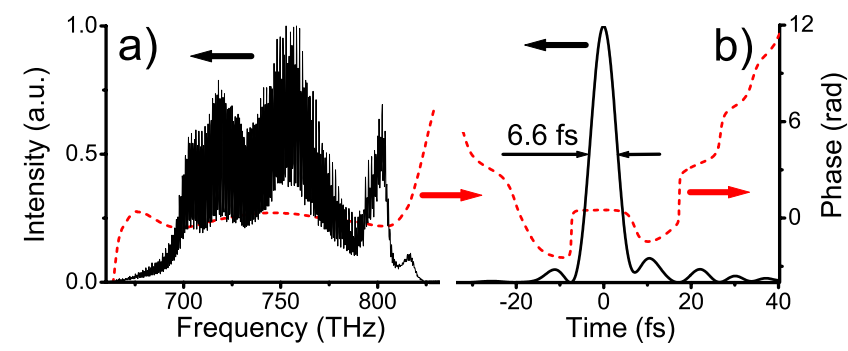

Fig. 3 The SPIDER measurement: (a) SPIDER interferogram (solid line) and reconstructed spectral pulse phase (dashed line); (b) reconstructed temporal pulse profile (solid line) and temporal phase (dashed line). The FWHM pulse duration is $6.6 \mathrm{fs}$.

this helps in bridging gaps in the detection of strongly modulated spectra with deep dips. The etalon is used at an oblique angle of $65^{\circ}$, resulting in a Fresnel reflectivity of $20 \%$ of each surface for s-polarization. This more symmetric beam splitting scheme enhances the efficiency of the setup by about an order of magnitude, as compared to earlier sub-10-fs SPIDER apparatus [19]. The etalon generates two replicas of the original pulse at a temporal distance of $\Delta \tau=1285 \mathrm{fs}$ in reflection, see Fig. 1. It should be noted that there is a small dispersion imbalance between the two replicas as one pulse has experienced an additional GDD of $10 \mathrm{fs}^{2}$ from the etalon material. This weak effect, however, is automatically taken into account by calibrating the apparatus with the spectral interferogram of the SH of the two replicas. The transmitted pulse is strongly chirped by a $10 \mathrm{~cm}$ long SF10 glass block with a group delay dispersion (GDD) of $15800 \mathrm{fs}^{2}$. An achromatic half wave retarder in the beam path of the chirped pulse rotates polarization for the broadband type-II interaction. All-reflective optics were used in the beam path of the two replicas. Mixing the three pulses in a type-II BBO crystal of $50 \mu \mathrm{m}$ thickness results in a shear of $13 \mathrm{THz}$ between the upconverted spectra of the two delayed replicas. The resulting SPIDER signal is dispersed in a $0.5 \mathrm{~m}$ spectrograph and detected by a fast 2048 tall-pixel line-scan CCD camera that covers a spectral range of $175 \mathrm{~nm}$ centered at a wavelength of $390 \mathrm{~nm}$.

In Fig. 3(a) the resulting interferogram (solid line) of the two spectrally sheared replicas is shown along with the retrieved spectral phase (dashed line) on the original frequency axis. The overall fringe visibility ranges from $670 \mathrm{THz}$ to $820 \mathrm{THz}$. This means that the spectral phase can be reconstructed over a bandwidth that hosts $98 \%$ of the pulse energy. For further interpretation the phase has to be shifted by $\Delta f=-365 \mathrm{THz}$ to match the fundamental spectral region, resulting in the reconstructed pulse shape shown in Fig. 3(b) with a FWHM of $6.6 \mathrm{fs}$. For completeness, the temporal phase is also shown in Fig. 3(b) as a dashed line. The temporal phase is essentially flat over the duration of the main pulse, but exhibits phase jumps between the satellites. The

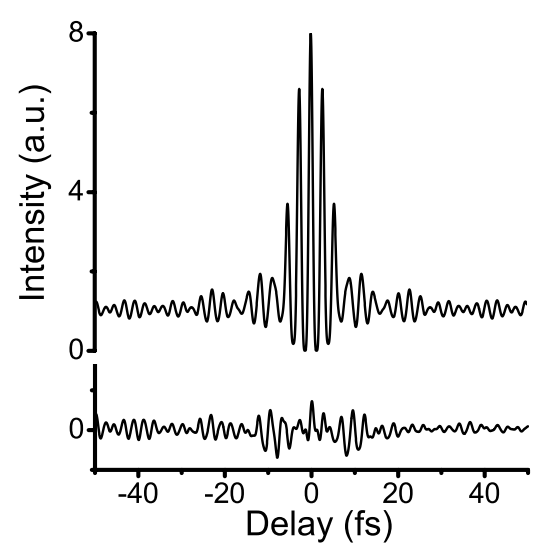

Fig. 4 Calculated interferometric autocorrelation from the SPIDER measurement (top) and difference to the measured IAC of Fig. 2 (bottom).

satellites in Fig. 3(b) are below $10 \%$ of the peak intensity, with three major satellites at $-11,+10$, and $+22 \mathrm{fs}$, which agrees with the satellite positions visible in the autocorrelations. The overall integrated satellite content amounts to $15 \%$ of the whole pulse. As a cross check, we compute the IAC from the pulse obtained by the SPIDER method and calculate the difference to the actually measured IAC, see Fig. 4. The overall agreement between the two IAC traces is very high, especially over the central 5 periods of the main pulse. In this range, the only measurable discrepancy arises from the 1:7.5 ratio of the measured trace. Moreover, SPIDER appears to measure slightly smaller satellites than apparent in the IAC, as can be seen from the higher difference around \pm 10 fs delay.

\section{The SEA-SPIDER method}

The SEA-SPIDER method was introduced recently by Kosik et al. [15] as a method that combines the virtues of spectral and (spatial) shearing interferometry. This method was further adapted for measuring few cycle pulses [16]. In this implementation, it includes the advantage of a zero-additional-phase (ZAP) measurement, similar to the ZAP-SPIDER [20], but can be made fully self-referenced, i.e., it does not rely on the wavelength calibration of a spectrograph. Due to fringe formation along the spatial coordinate of a dispersed image of the spectrometer entrance slit, this method works without the requirement of a small fringe periodicity for measuring strongly modulated spectra. Therefore a much lower spectral resolution is tolerable, which is particularly advantageous for single-shot measurements of broadband pulses. Of course, measuring and analyzing a two-dimensional SPIDER trace requires more time than in the one-dimensional methods, which makes SEA-SPIDER less suitable for high acquisition rates. On the other hand, the two-dimensionality turns out to 
be of advantage for monitoring space-time coupling effects. Moreover, the increased amount of data automatically leads to some statistical averaging when extracting the spectral phase information, which makes this method more robust. In contrast to the traditional SPIDER where two spectrally sheared replica pulses are sent collinearly into the spectrograph, SEA-SPIDER produces these two replicas in different directions. Imaging the overlapping focal points of the two replicas results in the dispersed spatial interference pattern of Fig. 5(a) due to a tilt between the wavefronts of the two pulses. The method gives an immediate picture of the spectral phase as a chirp on the pulse leads to a tilted interference pattern, i.e., the tilt of the fringes directly encodes the frequency dependent group delay, cf., Ref. [16].

The measurement of Fig. 5(a) is taken with the setup already described in Ref. [16]. SEA-SPIDER relies on the generation of three different pulses in mutually noncollinear paths. These three pulses are focused onto a single point in the nonlinear crystal. The actual pulse under test is a single replica of the original pulse that is reflected off an inconel-coated beam splitter. Except for the air path, no further dispersion affects this pulse. As in SPIDER, the pulse transmitted through the beam splitter is chirped using a $10 \mathrm{~cm}$ SF10 glass block. This pulse is further split into two in a Michelson type setup, which generates the two chirped pulses at a relative delay of $\Delta \tau=1550 \mathrm{fs}$, cf., Fig. 1. An adjustable delay between the two chirped pulses translates into a spectral shear between the two upconverted pulses when overlapping and focusing all three beams into a $50 \mu \mathrm{m}$ thick BBO crystal. To fulfill the broadband type II phase-matching condition we rotate the polarization of the chirped pulse by using a half wave retarder. Our measurement is taken with a spectral shear of $14 \mathrm{THz}$ between the pulses as could be verified by independently measuring the spectra of the two upconverted pulses. For SEA-SPIDER, self-consistent referencing can also be easily achieved. For determining the phase $\Delta k x$ introduced by the different propagation directions of the sheared pulses, the delay between the two chirped pulses has to be set to zero yielding an absolutely parallel fringe structure (not shown). This is analogous to the self-consistent calibration procedure of the regular SPIDER setup.

In the present configuration, the sensitivity of the SEA-SPIDER method is lower than that of the SPIDER apparatus described above. One reason for this is that the two-dimensional set-up generates a lower number of photons per pixel of the camera than in the onedimensional setup. A second concern are the losses of the metal-coated beam splitter mirrors. Nevertheless, the SEA-SPIDER interferogram of Fig. 5(a) was measured with a pulse energy of less than $2.5 \mathrm{~nJ}$. Inspecting Fig. 5(a), it can immediately be seen that the fringe structure is essentially flat, with only a slight tilt, which translates into a GDD of about $-15 \mathrm{fs}^{2}$. Except for the far wings of the spectrum, the fringe structure shows al-
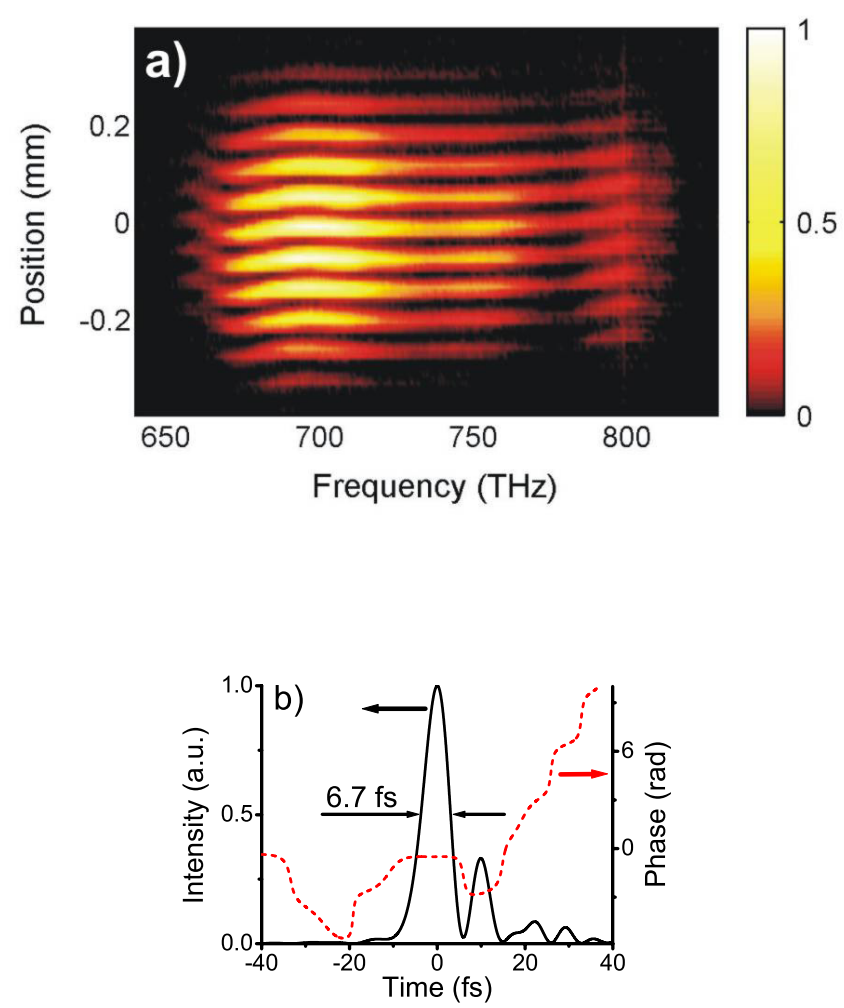

Fig. 5 Spatially encoded SPIDER (SEA-SPIDER) measurement: (a) SEA-SPIDER interferogram of the pulse. Deviation of the fringe structure from a frequency independent, pure parallel behavior encodes the group delay. (b) Reconstructed temporal pulse profile (solid line) and temporal phase (dashed line). The FWHM pulse duration is $6.7 \mathrm{fs}$.

most no curvature in the center, indicating the absence of higher-order dispersion. The resulting spectral phase is plotted as a dashed line in Fig. 10(a). This curve was extracted from 30 camera lines within the central part of the trace of Fig. 5(a) in the range from $x=-0.14$ to $+0.14 \mathrm{~mm}$.

The reconstruction of the pulse in the time domain is depicted in Fig. 5(b) as a solid line. For a direct comparison of the temporal pulse structure with the SPIDER result, we accounted for an approximately $50 \mathrm{~cm}$ longer beam path to the SPIDER by adding a GDD of 10 $\mathrm{fs}^{2}$ to the spectral phase retrieved by the SEA-SPIDER method, see Fig. 10(b). The temporal phase (dashed line) and the FWHM duration of 6.7 fs are practically identical to the results obtained by the SPIDER method. However, the satellite pulse at 10 fs delay appears more pronounced in SEA-SPIDER, reaching 30\% of the peak intensity. This results in a $10 \%$ higher energy content of the satellites compared to the SPIDER measurement. A comparison between the IAC calculated from the pulse measured by SEA-SPIDER and the measured IAC of Fig. 2 confirms that SEA-SPIDER is likely to have over- 


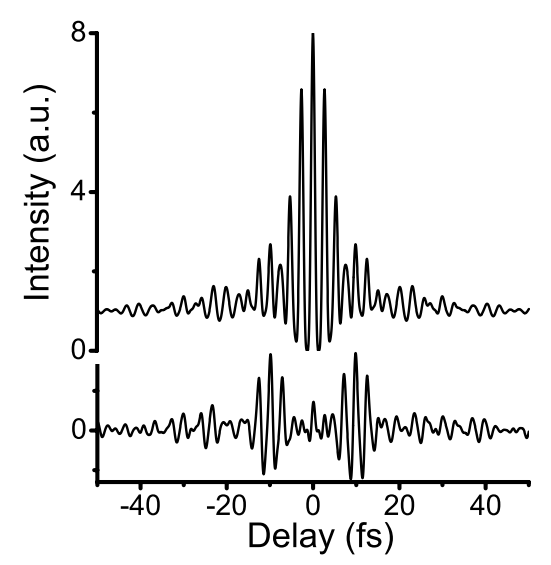

Fig. 6 Calculated interferometric autocorrelation from the SEA-SPIDER measurement (top) and difference to the measured IAC of Fig. 2 (bottom).

estimated the 10-fs satellite pulse, see Fig. 6. In addition, the IAC fringe structure of the satellite pulses at a delay of about $\pm 10 \mathrm{fs}$ (Fig. 6) appears to be essentially out of phase with the reference IAC measurements in Fig. 2. This gives rise to the strong difference signal around \pm 10 fs in Fig. 6 .

Except for these satellite pulses, the reconstruction appears essentially flawless for the central pulse. Comparing the reconstructed spectral phases, SEA-SPIDER certainly has an advantage as the traces exhibit clearly visible fringe patterns over an approximately $10 \%$ wider range than SPIDER. Therefore, the spectral phases measured by SEA-SPIDER are considered to be more reliable in the wings of the spectrum. We believe that the use of SEA-SPIDER may especially pay off when it comes to extremely broadband or strongly structured spectra. When comparing to the other methods, it has to be borne in mind that SEA-SPIDER clearly has the least reserve in terms of measuring smaller pulse energies. With our current setup we consider $\approx 2 \mathrm{~nJ}$ as a lower limit for the measurable pulse energy. Taking this into account, the measurements compare favorably to the other methods discussed here.

\section{Interferometric FROG}

Interferometric frequency-resolved optical gating (IFROG) can be considered as a combination of the interferometric autocorrelation (IAC) and FROG [14, $21,22]$. As the former it offers collinearity, the absence of geometrical smearing artifacts, and a larger amount of redundancy embedded in the interferometric trace. As a complete method it also offers the capacity to reconstruct the pulse shape and phase by iterative retrieval. The measurement setup and the data processing procedure are described in detail in Refs. [14] and [22]. Therefore only a brief description is given here. Our IFROG apparatus consists of a dispersion balanced
Michelson interferometer with two broadband (450 nm - $1050 \mathrm{~nm}$ ) $1 \mathrm{~mm}$ thick dielectric beam splitters for the generation of two collinearly propagating pulses with controlled temporal delay. These pulses are focused with a concave mirror of $10 \mathrm{~cm}$ focal length into a $10 \mu \mathrm{m}$ thick type-I BBO crystal. The second harmonic signal is isolated by a BG39 filter and dispersed in a $0.5 \mathrm{~m}$ spectrograph. A motorized translation stage is used in one arm of the interferometer, scanning the delay at a speed of about 20 to $40 \mathrm{fs}$ per second. The translation stage incorporates a rotary encoder that generates trigger pulses at a step width of 225 attoseconds, which are used to directly trigger a CCD camera at a line rate of 100 to $200 \mathrm{~Hz}$. This rapid acquisition on the fly prevents loss of fringe contrast due to interferometer drift and mechanical imperfections of the stage. The IFROG trace of the pulse under test is shown in Fig. 7(a).

As is clear from the IFROG trace in Fig. 7(a), IFROG produces an overwhelming amount of data in a broad spectral and temporal range. Sampling 2048 pixel spectra over a \pm 100 fs delay range results in some 2 million data points. Apart from the sheer number of data points, even small timing delay jitters in the correlator will lead to corrupt fringe structures, such that consistent reconstruction of the pulse shape is no longer possible. Therefore the data has to be reduced to a smaller grid, and the fringe structure has to be removed by Fourier filtering techniques. Similar to the structure of the IAC trace, the IFROG trace consists of an unmodulated part and two modulated components with a periodicity at the carrier frequency of the pulse and at its second harmonic $[14,21]$. Pulse retrieval can either be based on the unmodulated part of the signal, which consists of the regular SH-FROG trace plus a constant background term, or on the modulation at the fundamental frequency. In the following, we will only discuss the latter case as separation of the SH-FROG trace and the background term can be troublesome for experimental data and typically only yields a very limited dynamic range. The fundamental-modulation FROG (FM-FROG) trace, in contrast, is background-free, but has to be processed with special algorithms for pulse retrieval.

The isolated FM-FROG trace is displayed in Fig. 7(b). The data has been filtered out in the Fourier domain (with respect to the delay coordinate) and has then been transformed back into the delay representation. The FM-FROG trace has some peculiar properties, which differentiates it from other common FROG traces. First, it can be negative, which is indicated by blue regions in Fig. 7(b). Second, FM-FROG traces roll off much slower with increasing delay than SH-FROG traces. This is apparent in Fig. 7, as it is quite remarkable that the characterization of a sub-10-fs pulses results in clearly discernible structures at delays that are 20 times larger than the pulse duration. Effectively, this increases the sensitivity for reconstructing small satel- 

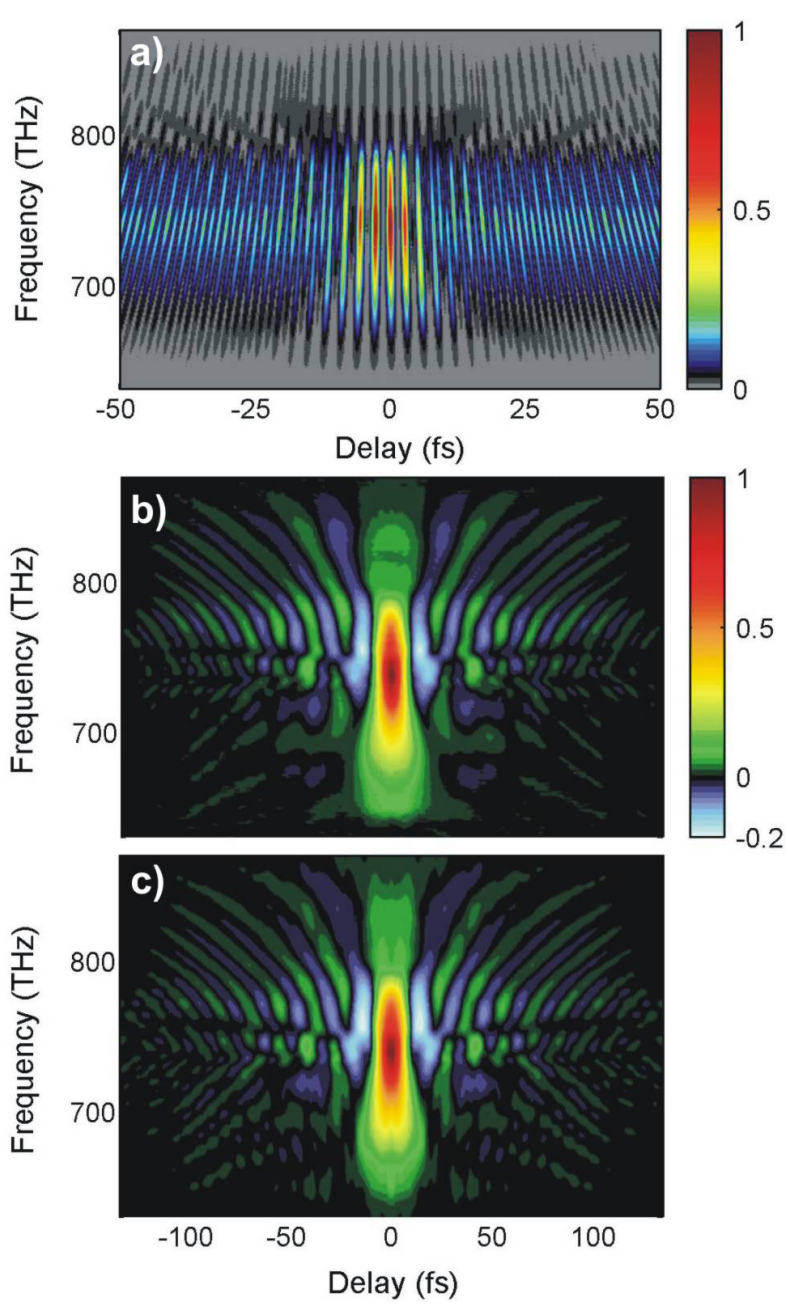

Fig. 7 Interferometric FROG measurement. (a) Central section of a fringe and frequency resolved interferometric autocorrelation measurement. (b) Phase-sensitively retrieved component of the IFROG trace at the modulation period of the fundamental wavelength (FM-FROG). (c) Iteratively reconstructed FM-FROG trace (original grid size: 256 pixels, FROG error: 0.004 after 500 iterations). Note that (b) and (c) are shown for a three times wider delay range than (a).

lites and the dynamic range of the method, as compared to other FROG variants.

We use a modified generalized projections algorithm $[22,23]$ to iteratively reconstruct the temporal intensity and phase profile of the pulse from the FMFROG measurement. The algorithm converges to the retrieved FM-FROG trace of Fig. 7(c) within 500 iterations. The resulting FROG error is 0.004 . The reconstruction is based on a $256 \times 256$ grid. The resulting pulse shape and spectrum are shown in Fig. 8. Figure 8(a) compares the reconstructed spectral power density (black solid line) to the measured spectral intensity (green data points). This frequency marginal check indicates consistency of the reconstruction process with independently measured data. Additionally, the spectral phase is plotted as a dashed line in Fig. 8(a). At first

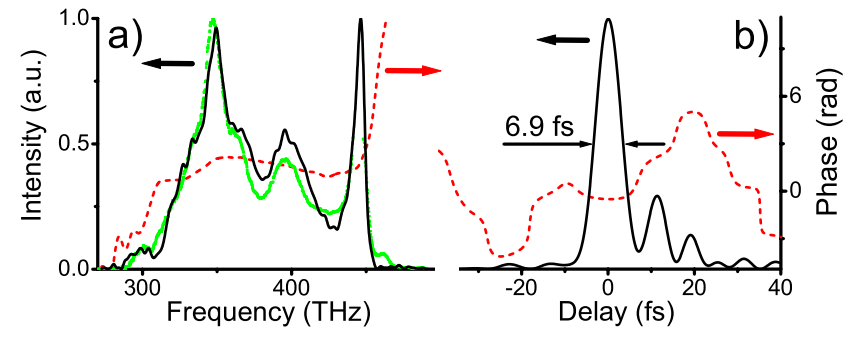

Fig. 8 Result of the FM-FROG retrieval routine: (a) retrieved spectral intensity (black solid line) and spectral phase (dashed line). The independently measured spectrum (green data points) is plotted for comparison. (b) Retrieved temporal pulse profile (solid line) and temporal phase (dashed line). The FWHM duration is 6.9 fs.

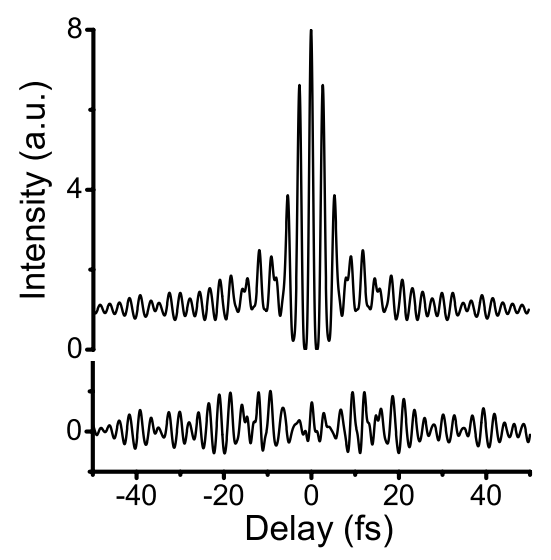

Fig. 9 Calculated interferometric autocorrelation from the FM-FROG retrieved pulse (top) and difference to the measured IAC of Fig. 2 (bottom).

glance, this data does not appear to compare well with the other methods, see Fig. 10(a). However, accounting for the beam splitter GDD of $1 \mathrm{~mm}$ of fused silica and for a $50 \mathrm{~cm}$ shorter air path compared to SPIDER, we need to subtract a total GDD of $25 \mathrm{fs}^{2}$. The resulting spectral phase [red dotted line in Fig. 10(b)] agrees very well with the results of the other two methods. Using this adjusted spectral phase, we calculated the resulting pulse shape in the time domain, see Fig. 8(b). Its duration is 6.9 fs (FWHM), essentially identical to the previous results.

The satellite structure of the pulse is similar to the results obtained by the SEA-SPIDER technique and dominated by two major satellites at +11 and at +19 fs. Their energy content is comparable to the SEA-SPIDER measurement but stronger than that measured with SPIDER and IAC. Therefore the IAC marginal in the satellite region is quite substantial and of the same order or even larger than the FM-FROG intensity itself. This indicates that the dominant contribution to the IAC marginal comes from phase differences of the satellite pulses between the two measurements, rather than from amplitude changes. Nevertheless the central main pulse 


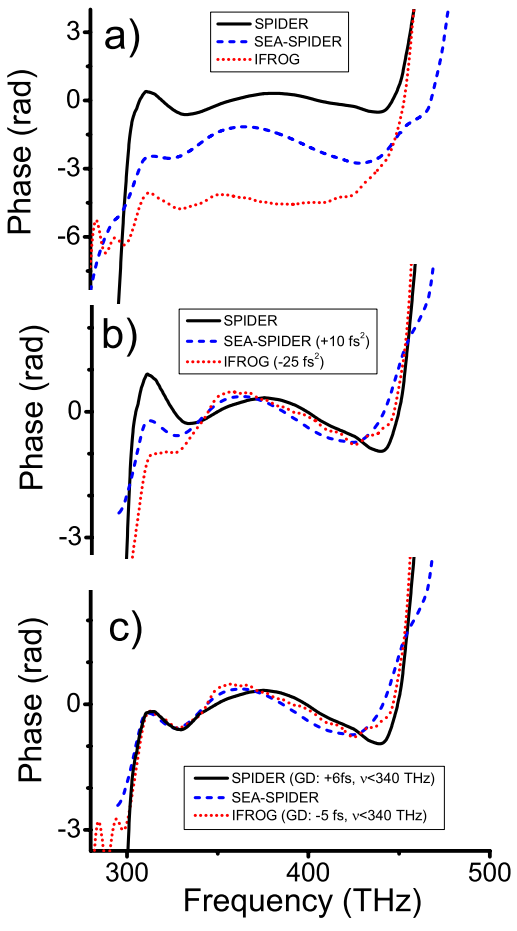

Fig. 10 Comparison of the spectral phases derived by the different methods: SPIDER (solid line), SEA-SPIDER (dashed line) and IFROG (dotted line). (a) Uncorrected data, obtained with laser optimized for shortest pulse duration measured with SPIDER; (b) spectral phases after correcting for different amounts of dispersion in the beam path, and (c) with additional adjustment of group delays below $340 \mathrm{THz}$. Pulse shapes in Figs. 3,5, and 8 have been computed with the spectral phases in (b).

is reconstructed with a comparable precision as with any of the other two methods reported before.

\section{Comparison}

The comparison of different characterization methods clearly reveals the virtues and limitations of current state-of-the-art laser pulse diagnostic techniques. The overall agreement between the measured pulse shapes and spectral phases is quite reassuring, indicating that either of the methods used in this work is suitable for characterizing few-cycle pulses. All methods, including the autocorrelation, yielded a pulse duration of $6.7 \pm 0.2$ fs, i.e., the measured pulse durations agree to within an error of about $5 \%$. The shapes of the reconstructed main pulse derived from either method appear to agree very well among each other and also show excellent IAC marginals. This indicates that either method is very well suited for characterizing the main pulse within a sequence of smaller satellite pulses. The durations derived from the individual methods generally reflect the trend seen in previous comparisons [24]. Again, the shortest pulse was measured by the IAC method.
This may go back to the assumption of a hyperbolic secant pulse shape, causing a systematic underestimation of pulse durations, and has been reported by many authors. A less biased evaluation of the IAC trace, though, did not reduce this tendency in our case. FROG has a certain tendency to measure a slightly longer pulse duration than the other methods, as was previously reported for another FROG variant [24]. We believe that this behavior may be caused by early stagnation of the retrieval algorithm, putting equal weight on the reconstruction of remote satellites as it does on the reconstruction of the main pulse.

The pulse duration is certainly one of the central concerns in advanced characterization. When optimizing laser systems, however, the spectral phase is probably even more important, as it directly shows the residual dispersion of a pulse. After accounting for the differential dispersion in the beam paths and beam splitters of the individual methods, we obtain the spectral phases shown in Fig. 10(b). For the central part of the spectrum, i.e. the range from 340 to $450 \mathrm{THz}$, the agreement between the different measurement methods is close to excellent, with rms deviations on the order of $0.2 \mathrm{rad}$. Outside this region, however, the three spectral phases tend to rapidly break away from each other. Comparing with the spectrum of Fig. 8, one realizes that the phases tend to diverge at steep spectral slopes close to the point where power densities are less than $30 \%$ of the peak value. It is striking that the spectral phases can be brought to close coincidence by adjusting the slope of the spectral phase, i.e. the group delay, of the SPIDER and IFROG phase below $340 \mathrm{THz}$ by +6 or $-5 \mathrm{fs}$, respectively. Taken for itself, the spectral pedestal region appears to be correctly characterized by either method. This is shown in Fig. 10(c), indicating that the major deviation between the measured data can be reduced to a single kink in the spectral phase, located at $340 \mathrm{THz}$.

There are certainly different ways to explain such findings. For example, the data have not been measured simultaneously, but on three subsequent days, albeit with an essentially identical laser spectrum. Therefore, a pronounced sensitivity of the long wavelength tail of the spectrum in respect to laser drift may hold as an explanation. Despite all care concerning laser stability this explanation cannot be totally ruled out. On the other hand, however, such phase kink artifacts have been described before as rather typical problems of pulse characterization methods when it comes to measuring strongly structured spectra, see e.g. Refs. $[9,10]$. Regardless of the characterization method used, spectral drops may cause an erroneous reconstruction of the relative group delay between two adjacent spectral regions. This uncertainty in measuring the relative timing between spectral pedestals and the main pulse results in the varying satellite structure between Figs. 3, 5, and 8. The width of the main pulse, in contrast, is mainly determined by the global curvature of the reconstructed spectral phase. 
Low-energy spectral pedestals only weakly affect this parameter. Extracting the curvature from the spectral phases by means of a weighted fit, we find that the group delay dispersion of the pulses agree to within $4 \mathrm{fs}^{2}$, i.e., a value that is compatible with the observed difference of the measured pulse duration of $5 \%$.

This discussion clearly pinpoints potentially problematic issues of all characterization methods. Even though the methods are fairly robust in terms of measuring the global group delay dispersion of a pulse and its duration, it is much more problematic to accurately measure the exact satellite structure of a pulse. This conclusion is backed up by inspection of the IAC marginals, Figs. 4, 6, and 9. Generally, looking only at the central part of the pulse, the agreement with the IAC trace is very good. At larger delays, however, the reconstructed satellites of the autocorrelation often appear out of phase with the IAC reference measurement and their amplitudes vary considerably for the different techniques used. In our comparison we actually observed the lowest overall IAC marginal for the pulse shape measured by regular SPIDER, whereas SEA-SPIDER shows a pronounced but isolated deviation at delays of $\pm 10 \mathrm{fs}$ but exhibits an even lower marginal otherwise. Interferometric FROG, in contrast, only yields excellent agreement close to zero delay, which is surprising to some extent, as one would expect it to be better suited for characterizing the satellite substructure of a pulse as this method is directly rooted in the time domain.

It should be noted that it is not a priori clear to what extent the IAC marginal is really a good test for an accurate satellite reconstruction. Other than in FROG, where numerous cross-checks guarantee the consistency of the data, IAC traces can contain undetected systematic errors, e.g., due to an insufficient phase-matching bandwidth of the nonlinear crystal. Such inconsistencies are more likely to occur in the spectral wings than in the center, which may particularly affect satellite pulses that are spectrally localized in the wings of the spectrum. We found indications for a limited conversion bandwidth in the IFROG measurements, using a BBO crystal of identical thickness, and corrected for it via the frequency marginal. Another issue may be caused by spatio-temporal coupling, as SEA-SPIDER is the only method that can reveal such artifacts whereas an IAC is totally insensitive towards it. Even though we averaged over the 5 most visible fringes when evaluating the spectral phase from Fig. 5(a), this still discards information in the wings of the beam profile and may explain some of the deviation seen in the marginal test above. This would indicate, e.g., that the trailing satellite has a different beam profile than the main pulse. As we were already close to the detection limit in our SEA-SPIDER measurements, however, a deeper spatially resolved analysis was not yet possible. Altogether the remaining differences between the reconstructed pulse shapes may appear weak, but based on our measurements neither of the methods can be attested a particular suitability for resolving the exact satellite structure of a complicated pulse. Therefore, if exact retrieval of the satellite structure is important, e.g. for pulse shaping experiments, application of cross-correlation based method such as XFROG [26] will certainly lead to better and more robust results. However, these methods require the presence of a short and unstructured reference pulse, which was not available in our case.

All aspects discussed so far concerned the accuracy of the individual methods. Even if a particular characterization method provides the most reliable result, it has to be discarded from many applications, e.g., when it only allows for one measurement per hour. We will therefore briefly address practicality as a further aspect of our comparison. In this regard, the IAC is certainly still the easiest to implement of all discussed methods. It is quite clear that IAC also delivers the weakest information on pulse shape and duration and only very coarse information on the spectral phase. From the complete methods, we currently consider the traditional SPIDER as the next simplest thing to do. Even though it may have its limitations in terms of spectral coverage and robustness against extremely modulated spectral shapes, the method is very fast, easy to align, and sensitive enough to measure rather weak pulses from an oscillator. This conclusion is certainly not overly fair, as we have spent a significant amount of time in optimizing our SPIDER setup, as compared to the IFROG and SEA-SPIDER implementations. We are confident that the latter two methods can be significantly improved in terms of practicality. The major issue with IFROG is currently the rather laborious reconstruction of the pulse via an iterative retrieval algorithm. Faster algorithms are under development, and direct non-iterative retrieval is currently discussed as an alternative that may completely resolve this issue [25]. Direct retrieval algorithms make IFROG an ideal add-on to an existing interferometric autocorrelator. While the autocorrelator suffices for day-to-day comparisons of a particular laser, more precise and reliable information could immediately be extracted by spectrally resolving the IAC traces. For improving our SEA-SPIDER setup, some more time has certainly to be put into further automating the necessary calibration procedures and for accelerating the extraction of pulse shapes from the SEA-SPIDER traces. Sensitivities are currently at the level of early implementations of sub-10-fs SPIDER setups [19], but may also benefit from optimized beam splitting schemes. These technical concerns put aside, however, SEA-SPIDER appears to be the method of choice when it comes to strongly structured spectra that exhibit deep dips and require an particularly robust method. SEA-SPIDER shows the widest coverage of the spectral phase far into the wings of the spectrum, and the intrinsic averaging over several phase measurements makes it superior to one-dimensional spectral interferometry methods. 


\section{Conclusion}

We have carefully characterized sub-7-fs pulses with four completely independent characterization techniques. All methods employed have been specifically optimized for use with extremely short pulses. In measuring the pulse duration and the global shape of the spectral phase, all methods deliver reliable information, with a quality of agreement between the individual methods that has not been reported before for such short pulse durations. Measured spectral phases agree within a GDD margin of $4 \mathrm{fs}^{2}$, which made it necessary to account for air path differences on the order of $10 \mathrm{~cm}$ when comparing to the experimental results. Altogether, no major discrepancy was observed when characterizing pulses of 2.5 optical cycles duration. A more detailed look at the reconstructed pulse shapes, however, clearly shows that reliable reconstruction of the satellite substructure is still difficult. This problem chiefly arises from strongly structured spectra, offering only weak support across low-intensity regions, which may cause phase-kink artifacts in the reconstructed pulse shapes. This artifact causes an erroneous reconstruction of the relative group delay between neighboring spectral regions, which will typically result in an incorrectly reconstructed satellite pulse. The occurrence of phase kinks is certainly not limited to spectral interferometry based methods, but can also appear in the retrieved pulse shapes of the FROG methods. Among all methods discussed, the redundant fringe structure of the SEA-SPIDER method appears to offer the best means for detecting and avoiding such phase kink artifacts. Similarly, one expects that FROG is less susceptible to such errors than SPIDER. In the light of our comparison, however, it seems that phase kink artifacts can arise with any method, and that there is currently no way of totally avoiding the possibility of their occurrence with strongly structured spectra. Apart from this issue, which is often of secondary nature in pulse characterization, all methods exhibited excellent performance, retrieving the pulse duration of a sub-7-fs pulse with a $5 \%$ relative uncertainty. Still, one has to be aware that there are certain limitations of these methods. Despite the limitations in the retrieval of satellite substructures, one can conclude that complete characterization methods have reached a maturity that allows their routine use in the challenging sub-10-fs region, delivering more information on pulse shaping, laser oscillators, and amplifiers than simple autocorrelation does.
3. R. Trebino, "Frequency-Resolved Optical Gating: The Measurement of Ultrashort Laser Pulses," Kluwer Academic Publishers, Boston, MA (2000).

4. C. Iaconis and I. A. Walmsley, Opt. Lett. 23, 792 (1998).

5. C. Iaconis and I. A. Walmsley, IEEE J. Quant. El. 35, 501 (1999).

6. G. Steinmeyer, D. H. Sutter, L. Gallmann, N. Matuschek, and U. Keller, Science 286, 1507 (1999).

7. G. Steinmeyer, J. Opt. A 5, R1 (2003).

8. J.-C. Diels, E. Van Stryland, and G. Benedict, Opt. Commun. 25, 93 (1978).

9. D. Keusters, H.-S. Tan, P. O'Shea, E. Zeek, R. Trebino, and W. S. Warren, J. Opt. Soc. Am. B 20, 2226 (2003).

10. G. Stibenz and G. Steinmeyer, Opt. Express 12, 6319 (2004).

11. A.-C. Tien, S. Kane, J. Squier, B. Kohler, and K. Wilson, J. Opt. Soc. Am. B 13, 1160 (1996).

12. A. Baltuška, M. S. Pshenichnikov, and D. A. Wiersma, IEEE J. Quant. El. 35, 459 (1999).

13. L. Gallmann, G. Steinmeyer, D. H. Sutter, N. Matuschek, and U. Keller, Opt. Lett. 25, 269 (2000).

14. G. Stibenz and G. Steinmeyer, Opt. Express 13, 2617 (2005).

15. E. M. Kosik, A. S. Radunsky, I. A. Walmsley, and C. Dorrer, Opt. Lett. 30, 326 (2005).

16. A. S. Wyatt, and I. A. Walmsley, G. Stibenz, and G. Steinmeyer, "Spatially Encoded Arrangement for Spectral Phase Interferometry for Direct Electricfield Reconstruction of sub-10-fs pulses," submitted to Opt. Lett. (2005).

17. K. Naganuma, K. Mogi, and H. Yamada, IEEE J. Quantum Electron. 25, 1225 (1989).

18. J.-H. Chung, and A. M. Weiner, IEEE J. Sel. Top. Quantum Electron. 7, 656-666 (2001).

19. L. Gallmann, D. H. Sutter, N. Matuschek, G. Steinmeyer, U. Keller, Appl. Phys. B 70, S67 (2000).

20. P. Baum, S. Lochbrunner, and E. Riedle, Opt. Lett. 29, 210 (2004).

21. I. Amat-Roldán, I. G. Cormack, P. Loza-Alvarez, E. J. Gualda, and D. Artigas, Opt. Express 12, 1169 (2004).

22. G. Stibenz and G. Steinmeyer, "Structures of Interferometric frequency-resolved optical gating," submitted to IEEE J. Sel. Top. Quantum Electron. (2005).

23. K. W. DeLong, D. N. Fittinghoff, R. Trebino, B. Kohler, K. Wilson, Opt. Lett. 19, 2152 (1994).

24. L. Gallmann, D. H. Sutter, N. Matuschek, G. Steinmeyer, U. Keller, Appl. Phys. B 70, S67 (2000).

25. I. Amat-Roldán, I. G. Cormack, P. Loza-Alvarez, D. Artigas Opt. Lett. 30, 1063 (2005).

26. S. Linden, H. Giessen, J. Kuhl, Phys. Stat. Solid. B 206, 119 (1998).

\section{References}

1. D. J. Kane and R. Trebino IEEE J. Quantum Electron. 29571 (1993).

2. R. Trebino, K. W. De Long, D. N. Fittinghoff, J. N. Sweetser, M. A. Krumbügel, B. A. Richman, and D. J. Kane, Rev. Sci. Instrum. 68, 3277 (1997). 